\title{
What is the Latest on Catheter Ablation of Atrial Fibrillation?
}

Jason G. Andrade MD, Marc W. Deyell MD MSc, Laurent Macle MD DOI: $10.22374 /$ cjgim.v13iSP1.308

\begin{abstract}
About the Authors
Jason Andrade and Laurent Macle are with the Electrophysiology Service at the Montreal Heart Institute and the Department of Medicine, Université de Montréal, Montreal, Canada. Marc Deyell with the Heart Rhythm Services, Department of Medicine, The University of British Columbia, British Columbia, Canada.

Correspondence may be directed to: Imacle@mac.com
\end{abstract}

Atrial fibrillation (AF) is the most common sustained cardiac arrhythmia observed in clinical practice, affecting $1-2 \%$ of the general population. AF is associated with reductions in quality of life, functional status, cardiac performance, and survival. ${ }^{1}$ In addition, AF accounts for $1.0-2.7 \%$ of total annual healthcare expenditures, with a sizeable proportion of these expenses attributed to direct costs associated with emergency department visits and acute care hospitalization. ${ }^{2-5}$

The contemporary management of AF is centred on symptomatic improvement, reduction in healthcare utilization, and reduction in AF-associated morbidity and mortality (in particular the prevention of stroke or systemic thromboembolism). ${ }^{6}$ While ventricular rate control can be effective, many patients remain symptomatic despite optimal rate control. For these patients, restoration and maintenance of sinus rhythm can alleviate symptoms, and improve quality of life. Oral antiarrhythmic drug (AAD) therapy, which remains the "first-line" therapy for the maintenance of sinus rhythm, has only modest efficacy at maintaining sinus rhythm over the long term and can be associated with significant side-effects such as pro-arrhythmia, heart failure, and organ toxicity. ${ }^{7-12}$

For many patients, catheter ablation offers an alternative for maintaining sinus rhythm when AADs have been ineffective or cannot be tolerated. While catheter ablation has not been proven to improve mortality, it has been shown to be universally superior to AADs for the maintenance of sinus rhythm (AF elimination in $66-89 \%$ with catheter ablation vs. $9-58 \%$ with AAD). In addition, catheter ablation significantly improves symptoms, exercise capacity, and quality of life. ${ }^{13-23}$
The focus of this article will be a brief review of the history of AF ablation, followed by a discussion of four areas where significant recent developments have occurred.

\section{Evolution of Current Ablation Strategies and Techniques}

Up until the mid to late 1980s the "multiple wavelet hypothesis" was the dominant mechanistic theory of AF pathophysiology. This theory postulated that AF results from the presence of multiple independent coexisting wavelets that are occurring simultaneously and propagating randomly throughout the atria. This hypothesis postulated that AF could be initiated and then perpetuated indefinitely as long as the atrium had a sufficient area with adequately short refractory periods. As such, the early surgical and percutaneous interventions aimed to decrease arrhythmia perpetuation by compartmentalizing the atrium into smaller regions. It was thought that reducing the excitable mass of atrial tissue would render the atria incapable of sustaining the critical number of circulating wavelets. The approach was associated with only moderate success.

In the late 1990s Haïssaguerre and colleagues demonstrated that $\mathrm{AF}$ was a triggered arrhythmia initiated by rapidly repetitive discharges predominantly originating from the pulmonary veins (PVs). This led to the development of percutaneous procedures designed to electrically isolate the PV from the left atrium. Over the past 20 years the technique of PV isolation (PVI) has evolved significantly from focal ablation of discrete triggers within the $\mathrm{PV}$, to circumferential ablation of the left atrial myocardium outside of the tubular veins with a goal of large circumferential 


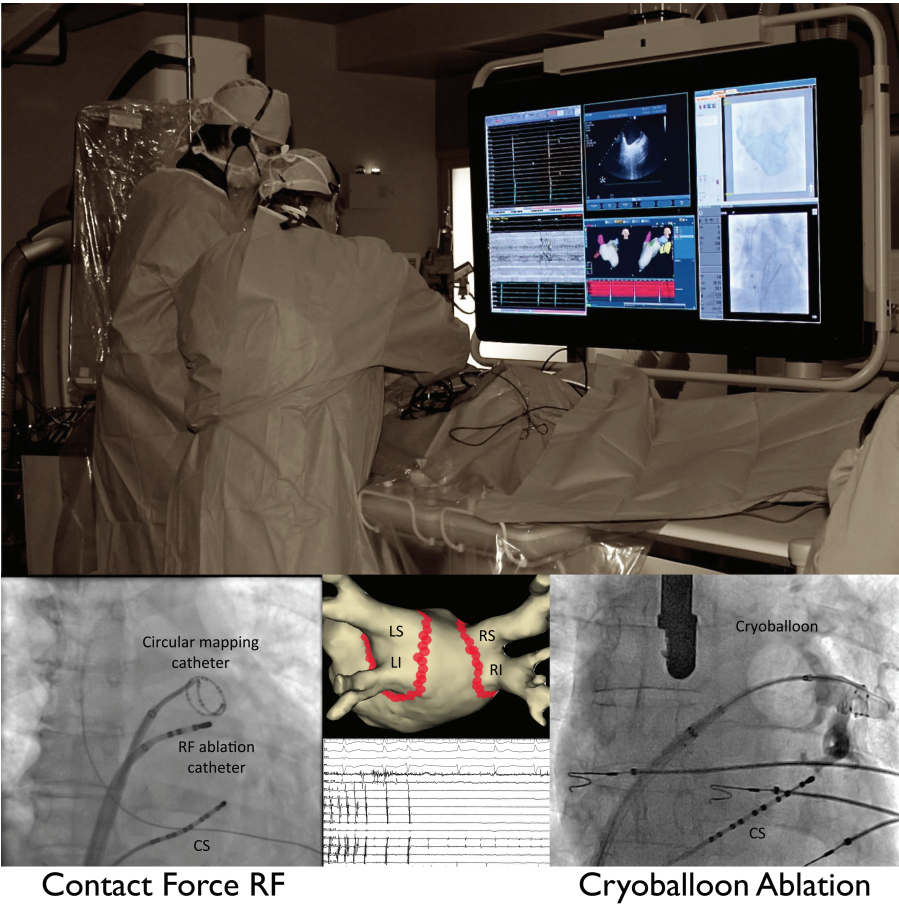

Figure 1. Example of AF ablation with point-by-point contact force radiofrequency (bottom left) and cryoballoon ablation (bottom right).

electrical PVI (i.e., electrical conduction block into and out of the PVs). This contemporary approach not only targets the initiating triggers of $\mathrm{AF}$ (the PVs) but also the mass of electrically active LA tissue capable of perpetuating AF (Figure 1).

Unfortunately, although the results of catheter ablation are unequivocally superior to antiarrhythmic drug therapy, they are not perfect. It can be anticipated that only approximately $70 \%$ of paroxysmal AF patients will remain arrhythmia- and AADfree after a single ablation procedure. For persistent AF patients the progressive pathoanatomical changes in the atria result in a further reduced procedural efficacy (50-60\% vs. 66-89\% in paroxysmal AF), but additional ablation strategies targeting the substrate outside the PVs (linear ablation or ablation of complex atrial fractionated electrograms) are not superior to PVI alone. ${ }^{24}$ Therefore, PVI remains the cornerstone of catheter ablation for paroxysmal and persistent $\mathrm{AF}^{25}$

However, it is important to realize that even in the face of arrhythmia recurrence a clinical improvement can be obtained. Recent studies have suggested that complete elimination of AF may not be necessary, and that significant clinical benefit can be obtained if the overall arrhythmia burden (i.e. time in AF) is reduced. For example, a recent analysis from the CASTLE-AF trial demonstrated that a $50 \%$ reduction in $\mathrm{AF}$ burden was associated with a significant reduction in the risk of death (HR 0.30), or heart failure hospitalization (HR 0.43). Therefore, although the arrhythmia might not be considered "cured" in all patients, a significant proportion of patients derive meaningful clinical benefit.

\section{Novel Technologies to Ensure Durable Pulmonary Vein Isolation}

While electrical PVI may be achieved acutely, the creation of durable scar around the PVs with a percutaneous procedure is challenging. With time, inadequate ablation leads to recovery of electrical conduction between the PV and LA, which may trigger recurrent arrhythmia episodes. In this regard several novel ablation technologies have been designed in an effort to improve ablation lesion creation.

\section{Contact Force Sensing Catheters}

Contact force (CF) sensing is a newly developed technology embedded within the radiofrequency ablation catheter that allows for the real-time estimation of the contact between the tip of the catheter and the target myocardium. Durable lesion formation in the atrium requires adequate catheter electrode-tissue contact. Insufficient tissue contact can result in edema with only transient loss of conduction. Available data suggests that incorporating real-time $\mathrm{CF}$ assessment into the ablation procedure results in a reduction in procedure time, ablation time, and total energy delivery. ${ }^{26,27}$ Recent studies suggested improved arrhythmia outcomes when the procedure was performed with adequate CF parameters (84\% one-year freedom from $\mathrm{AF}$ in the $47 \%$ of patients in whom ablation was in the target range $\geq 80 \%$ of the time in SMART-AF, and $76 \%$ one-year freedom from AF in the $57 \%$ of patients in whom $\geq 90 \%$ of the lesions were $>10 \mathrm{~g}$ in TOCCASTAR). ${ }^{28,29}$

\section{Balloon-Based Technologies}

While focal point-by-point radiofrequency catheter ablation has shown considerable success, the procedure is time-consuming and dependent on operator competency. ${ }^{30}$ In response considerable effort has been directed towards the development of technologies that are less reliant on operator dexterity. The most mature of these balloon-based technologies is the Cryoballoon (Medtronic, Minneapolis, MN), a purpose-built ablation catheter specifically designed for PVI. Instead of the "heat" utilized with radiofrequency ablation, the cryoballoon employs freezing inside the balloon to destroy the atrial tissue. When compared to radiofrequency cryothermal energy offers several potential advantages, including improved catheter stability (due to tissue adherence at freezing temperatures), minimal endocardial surface disruption, reduced thrombogenicity, and preserved ultrastructural tissue integrity leading to a lower perforation risk. ${ }^{31}$ Balloon-based technologies 
continue to evolve and recent studies have demonstrated improved arrhythmia-free survival with the second-generation cryoballoon compared to the first generation cryoballoon (OR of arrhythmia recurrence 0.34 [0.26-0.45]; 10 studies, 2310 patients). ${ }^{32,33}$ While these outcomes are comparable to standard radiofrequency ablation, ${ }^{34,35}$ ongoing studies are evaluating the effectiveness of 2 nd generation cryoballoon ablation compared to $\mathrm{CF}$ sensing radiofrequency ablation. ${ }^{36}$

Other balloon-based technologies include: (1) the HeartLight laser balloon (CardioFocus, Marlborough, MA), which is an endoscopic laser ablation catheter that allows direct visualization of the endocardial surface of the PV during infrared light energy ablation; (2) The multi-electrode Helios radiofrequency balloon (Biosense); and (3) The Apama radiofrequency Balloon (Boston Scientific, Marlborough, USA). Each of these technologies have recently published encouraging short-term safety and efficacy outcomes, with large randomized multicenter studies ongoing.

\section{Ablation as a First-Line Treatment}

The ideal management of patients with newly diagnosed symptomatic AF remains unknown. Current practice guidelines recommend a trial of antiarrhythmic drugs prior to considering an invasive ablation procedure. However, the universal superiority of ablation over antiarrhythmic drug therapy suggests that performing catheter ablation earlier may offer an opportunity not only for better symptom control but also to halt the progressive pathophysiological and anatomical changes associated with $\mathrm{AF}^{37}$ So far the evidence supporting "first-line" catheter ablation (i.e., as an initial therapy prior to AAD) is promising, but far from definitive. The MANTRA-PAF Study and the RAAFT studies randomized patients to either first-line ablation or first-line AADs. ${ }^{14,38,39}$ Despite disparate ablation techniques, these studies collectively demonstrated an improved freedom from recurrent arrhythmia (37\% reduction in AF recurrence vs. AAD therapy), an improved freedom from symptomatic AF (43\% reduction in symptomatic AF vs. AAD therapy), and a reduction in the overall AF burden ( $50 \%$ reduction over AAD therapy). While the results of these studies suggest that ablation is more effective than AAD therapy as first-line treatment, a significant proportion of patients in the intervention group experienced arrhythmia recurrence. Thus, the issue of first-line ablation remains an open question, where further research is required before first-line catheter ablation can be considered routine for most patients with symptomatic paroxysmal AF.

\section{Ablation in Patients with Heart Failure}

$\mathrm{AF}$ and heart failure (HF) are global cardiovascular epidemics. These conditions frequently coexist, and are both increasing in prevalence. ${ }^{40,41} \mathrm{AF}$ is an independent predictor of progression, hospitalization and death in the HF population. ${ }^{42-46}$ While restoring and maintaining sinus rhythm may be a therapeutic target to improve outcomes, large randomized controlled trials of antiarrhythmic drugs (AAD) have failed to support this hypothesis. ${ }^{47-51}$ It is postulated that the attenuated benefit observed with AADs therapy is related to cardiac and non-cardiac toxicities (e.g., pro-arrhythmia, conduction block, negative inotropy), and ablation may be a more efficacious means to improve outcomes.

To date seven randomized trials have been performed, in addition to an observational meta-analysis, and an observational multi-centre cohort study. Collectively these studies have demonstrated a single procedure success (e.g., elimination of any AF episodes $>30$ seconds) in the range of $40-69 \%$, with multiple procedures improving the success up to $88 \%$. Beyond arrhythmia recurrence, catheter ablation of AF in HF patients with LV systolic dysfunction appears to be associated with improvement in left ventricular ejection fraction (LVEF improvement of 4.5-18\%), exercise performance ( $\mathrm{VO} 2 \mathrm{max}$ improvement of $3 \mathrm{~mL} / \mathrm{kg} / \mathrm{min}$ vs. comparator, and 6-minute walk improvement of 20-70 meters from baseline), and quality of life (33\% average improvement in Minnesota Living with HF Questionnaire score). ${ }^{52-58}$

However, a truer measure of the utility of catheter ablation in patients with $\mathrm{AF}$ and $\mathrm{HF}$ with reduced systolic function is the objective outcomes, such as mortality and hospitalization. While a recent large RCT failed to demonstrate significant mortality benefit in unselected populations, two recent randomized trials have demonstrated that catheter ablation of AF in HF patients with reduced systolic function population results in significant improvement in all-cause mortality as well as fewer HF hospitalizations. ${ }^{52,58,59}$ The first of these studies was the AATAC study, which randomized 203 patients with New York Heart Association functional class II to III HF and an LVEF $<40 \%$ to catheter ablation $(\mathrm{n}=102)$ or amiodarone rhythm control $(n=101) .{ }^{52}$ After 24-27 months of follow-up patients in the ablation group had a significantly greater freedom from recurrent $\mathrm{AF}$ ( $70 \%$ vs. $34 \% ; \mathrm{P}<0.001)$. In addition, the secondary endpoints of unplanned hospitalization and all-cause mortality were both significantly reduced ( $45 \%$ and $56 \%$ respectively), corresponding to a NNT of 3.8 for unplanned hospitalization and 10 for all-cause mortality. The second study, CASTLE-AF, randomized patients with symptomatic paroxysmal or persistent AF, New York Heart Association class II-IV HF, and an LVEF $\leq 35 \%$ to catheter ablation (179 patients) or medical therapy (184 patients). ${ }^{58}$ All patients had a cardiac implantable device (ICD or CRT-ICD). After a median follow-up of 37.8 months, patients in the ablation group were significantly less likely to meet the primary composite end point of all-cause mortality or HF 
admission (16.1\% absolute reduction; HR 0.62; 95\% CI 0.43-0.87; $\mathrm{p}=0.006$ ). Similar to AATAC, there was a $47 \%$ relative reduction in all-cause mortality (HR 0.53; 95\% CI 0.32-0.86) and a 44\% reduction in HF hospitalization (HR 0.56; 95\% CI 0.37-0.83).

The third study was CAMERA-MRI, which evaluated catheter ablation vs. medical rate control in 68 patients with persistent $\mathrm{AF}$ and left ventricular systolic dysfunction (LVEF $\leq 45 \%) .{ }^{57}$ AF burden was assessed in ablation patients via implanted loop recorder, and serial holter monitors in the medical rate control patients. The primary endpoint was change in the LVEF on magnetic resonance imaging (MRI) at six months. The authors observed an $18 \pm 13 \%$ absolute improvement in the ablation arm vs. $4.4 \pm 13 \%$ in the rate control arm $(\mathrm{P}<0.0001)$. The absence of ventricular scar, as defined by late gadolinium enhan cement on cardiac MRI, predicted greater improvement in LVEF (22.3\% vs. $11.6 \%$ in those with scar, $\mathrm{p}=0.0069)$, and a greater likelihood of LVEF normalization ( $73 \%$ vs. $21 \%$ in those with scar, $\mathrm{P}=0.009$ ). As such, it is possible that MRI may be used to prospectively identify HF patients with reduced systolic function who may benefit from catheter ablation of AF.

Taken together these studies demonstrate that catheter ablation is associated with an improvement in ejection fraction and clinical outcomes when compared to medical therapy. Most striking is the observation that these adverse outcomes seem to be more linked to the overall AF burden. Specifically, the clinical benefit observed in CASTLE-AF was not dependent on the complete elimination of AF. Notably, while $63.1 \%$ of patients in the ablation group and $21.7 \%$ of patients in the medical therapy group were free of recurrence at the 60-month follow-up visit, the time in $\mathrm{AF}$ was reduced to $\sim 25 \%$ in the ablation group compared to $\sim 60 \%$ in the medical therapy group. A subsequent analysis of these patients demonstrated an AF burden $<5 \%$ was associated with a more than 3 times significantly greater freedom from all-cause mortality or HF hospitalization at 1 year, when compared to greater burdens of AF. It is hypothesized that the reduction in AF burden facilitates improvement in cardiac hemodynamic function (including cardiac output), autonomic nervous system performance, and reverse remodeling, which could also explain the lack of benefit observed in the rate control group in CAMERA-MRI.

\section{Lifestyle Intervention to Target AF Perpetuating Substrate}

AF is a complex chronic cardiovascular condition, with modifiable lifestyle and cardiovascular risk factors impacting the risk for AF development, AF progression, and AF recurrence after cardioversion and catheter ablation. Modifiable risk factors such as hypertension, obstructive sleep apnea, obesity, physical

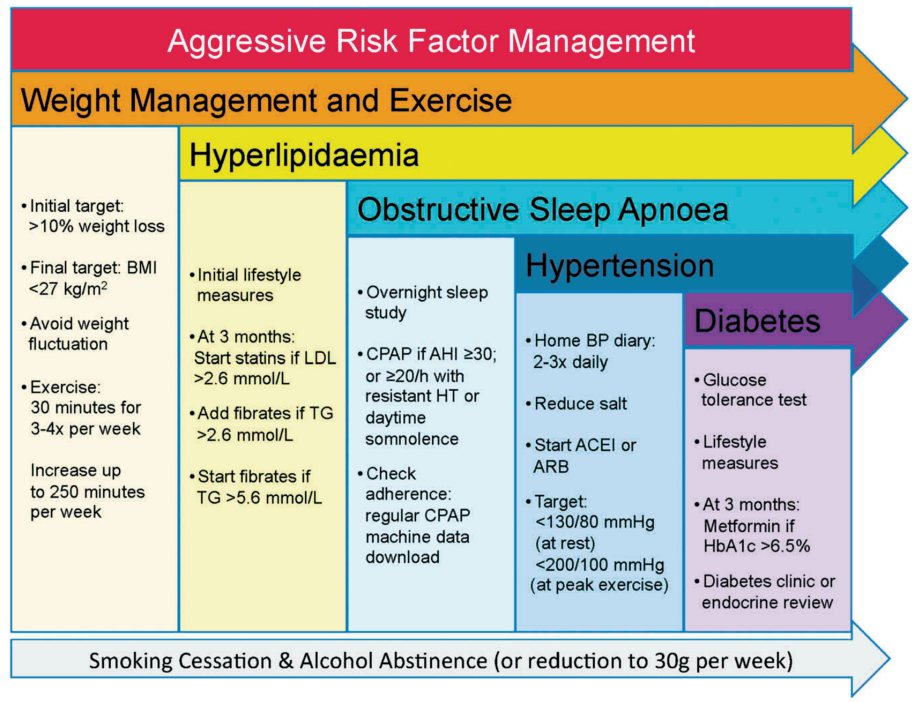

Figure 2. Risk factor management targets and strategies from the ARREST-AF cohort study. (ACEI - angiotensin converting enzyme inhibitor; AHI - apneahypopnea index; ARB - angiotensin receptor blocker; BP - blood pressure; CPAP - continuous positive airway pressure; LDL - low density lipoprotein; TG - triglycerides). Reproduced with permission from Lau et al. ${ }^{60}$

inactivity, alcohol and tobacco use, and diabetes mellitus have all been shown to significantly contribute to AF frequency, duration, and symptom severity. As such, interventions to optimally manage these comorbidities have been postulated to improve outcomes.

The ARREST-AF cohort study examined 149 patients with a body mass index $\geq 27 \mathrm{~kg} / \mathrm{m} 2$ and $\geq 1$ cardiac risk factors, dividing them into 61 patients who opted for risk factor management (RFM) and 88 control subjects (Figure 2). In the RFM group: blood pressure was treated to a target of $<130 / 80 \mathrm{mmHg}$ (ACEI/ $A R B$ preferred), weight management targeted a $>10 \%$ weight loss to a $\mathrm{BMI}<27 \mathrm{~kg} / \mathrm{m} 2$, exercise was targeted to start at 90 minutes per week increasing to 250 minutes per week, obstructive sleep apnea (AHI $\geq 30$ ) was treated with CPAP therapy, lipids were treated to contemporary targets, with elevated fasting blood sugar/ $\mathrm{HbAlc}$ treated with metformin to target an $\mathrm{HbAlc}<7 \%$. At a mean follow-up of $\sim 42$ months post ablation the RFM groups achieved greater reductions in weight $(-13.2 \pm 5.4 \mathrm{~kg}$ vs. $-1.5 \pm 5.1$ $\mathrm{kg}$; $=0.002)$, blood pressure ( $34.1 \pm 7.5 \mathrm{mmHg}$ vs. $20.6 \pm 3.2 \mathrm{mmHg}$; $\mathrm{p}=0.003$ ), glycemic control (HbAlc levels $<7 \%$ in $100 \%$ vs. $29 \%$; $\mathrm{p}=0.001$ ), and lipid values (controlled in $46.2 \%$ vs. $17 \%$; $\mathrm{p}=0.01$ ). Arrhythmia-free survival rates after catheter ablation were $87 \%$ with RFM compared with $18 \%$ for the control group $(\mathrm{p}<0.001)$, with a significantly greater reduction in symptom scores and global well-being $(\mathrm{p}<0.001)$. 


\section{Conclusion}

Catheter ablation is an effective treatment for AF. Pulmonary vein isolation remains the cornerstone of $\mathrm{AF}$ ablation procedures, with considerable effort having been directed towards developing technologies to achieve safer and more durable lesions. Ongoing research is being invested in studying ablation strategies to improve outcomes in various AF populations.

\section{References}

1. Wolf PA, Mitchell JB, Baker CS, et al. Impact of AF on mortality, stroke, and medical costs. Arch Intern Med 1998;158(3):229-34.

2. Andrade J, Khairy P, Dobrev D, Nattel S. The clinical profile and pathophysiology of AF: relationships among clinical features, epidemiology, and mechanisms. Circ Res 2014;114(9):1453-68.

3. Stewart S, Murphy NF, Walker A, et al. Cost of an emerging epidemic: an economic analysis of AF in the UK. Heart 2004;90(3):286-92.

4. Wu EQ, Birnbaum HG, Mareva M, et al. Economic burden and comorbidities of AF in a privately insured population. Current medical research and opinion 2005;21(10):1693-9.

5. Reynolds MR, Essebag V, Zimetbaum P, et al. Healthcare resource utilization and costs associated with recurrent episodes of AF: the FRACTAL registry. J Cardiovasc Electrophysiol 2007;18(6):628-33.

6. Gillis AM, Verma A, Talajic M, et al. Canadian Cardiovascular Society AF guidelines 2010: rate and rhythm management. Can J Cardiol 2011;27(1):47-59.

7. Roy D, Talajic M, Dorian P, et al. Amiodarone to prevent recurrence of AF. Canadian Trial of AF Investigators. N Engl J Med 2000;342(13):913-20.

8. Singh SN, Singh BN, Reda DJ, et al. Comparison of sotalol versus amiodarone in maintaining stability of sinus rhythm in patients with AF (Sotalol-Amiodarone Fibrillation Efficacy Trial [Safe-T]). Am J Cardiol 2003;92(4):468-72.

9. Roden DM. Mechanisms and management of proarrhythmia. Am J Cardiol 1998;82(4A):49I-57I.

10. Doyle JF, Ho KM. Benefits and risks of long-term amiodarone therapy for persistent AF: a meta-analysis. Mayo Clin Proc 2009;84(3):234-42.

11. Nademanee K, Schwab MC, Kosar EM, et al. Clinical outcomes of catheter substrate ablation for high-risk patients with AF. J Am Coll Cardiol 2008;51(8):843-9.

12. Corley SD, Epstein AE, DiMarco JP, et al. Relationships between sinus rhythm, treatment, and survival in the Atrial Fibrillation Follow-Up Investigation of Rhythm Management (AFFIRM) Study. Circulation 2004;109(12):1509-13.

13. Pappone C, Augello G, Sala S, et al. A randomized trial of circumferential pulmonary vein ablation versus antiarrhythmic drug therapy in paroxysmal AF: the APAF Study. J Am Coll Cardiol 2006;48(11):2340-7.

14. Wazni OM, Marrouche NF, Martin DO, et al. Radiofrequency ablation vs antiarrhythmic drugs as first-line treatment of symptomatic AF: a randomized trial. JAMA 2005;293(21):2634-40.

15. Jais P, Cauchemez B, Macle L, et al. Catheter ablation versus antiarrhythmic drugs for AF: the A4 study. Circulation 2008;118(24):2498-505.

16. Oral H, Pappone C, Chugh A, et al. Circumferential pulmonary-vein ablation for chronic AF. N Engl J Med 2006;354(9):934-41.

17. Packer DL, Irwin JM, Champagne J, et al. Cryoballoon ablation of pulmonary veins for paroxysmal atrial fibrillation: first results of the North American Arctic Front STOP-AF pivotal trial. J Am Coll Cardiol 2010;55:E3015-6.

18. Krittayaphong R, Raungrattanaamporn O, Bhuripanyo K, et al. A randomized clinical trial of the efficacy of radiofrequency catheter ablation and amiodarone in the treatment of symptomatic AF. Journal of the Medical Association of Thailand = Chotmaihet thangphaet 2003;86 Suppl 1:S8-16.
19. Stabile G, Bertaglia E, Senatore G, et al. Catheter ablation treatment in patients with drug-refractory AF: a prospective, multi-centre, randomized, controlled study (Catheter Ablation For The Cure Of AF Study). Eur Heart J 2006;27(2):216-21.

20. Wilber DJ, Pappone C, Neuzil P, et al. Comparison of antiarrhythmic drug therapy and radiofrequency catheter ablation in patients with paroxysmal AF: a randomized controlled trial. JAMA 2010;303(4):333-40.

21. Bunch TJ, Crandall BG, Weiss JP, et al. Patients treated with catheter ablation for AF have long-term rates of death, stroke, and dementia similar to patients without AF. J Cardiovasc Electrophysiol 2011;22(8):839-45.

22. Piccini JP, Lopes RD, Kong MH, et al. Pulmonary vein isolation for the maintenance of sinus rhythm in patients with AF: a meta-analysis of randomized, controlled trials. Circ Arrhythm Electrophysiol 2009;2(6):626-33.

23. Wokhlu A, Monahan KH, Hodge DO, et al. Long-term quality of life after ablation of AF the impact of recurrence, symptom relief, and placebo effect. J Am Coll Cardiol 2010;55(21):2308-16.

24. Verma A, Jiang CY, Betts TR, et al. Approaches to catheter ablation for persistent AF. N Engl J Med 2015;372(19):1812-22.

25. Calkins H, Hindricks G, Cappato R, et al. 2017 HRS/EHRA/ECAS/APHRS/ SOLAECE expert consensus statement on catheter and surgical ablation of AF. Europace 2018;20(1):e1-e160.

26. Reddy VY, Shah D, Kautzner J, et al. The relationship between contact force and clinical outcome during radiofrequency catheter ablation of AF in the TOCCATA study. Heart Rhythm 2012;9(11):1789-95.

27. Neuzil P, Reddy VY, Kautzner J, et al. Electrical reconnection after pulmonary vein isolation is contingent on contact force during initial treatment: results from the EFFICAS I study. Circ Arrhythm Electrophysiol 2013;6(2):327-33.

28. Reddy VY, Dukkipati SR, Neuzil P, et al. Randomized, Controlled Trial of the Safety and Effectiveness of a Contact Force-Sensing Irrigated Catheter for Ablation of Paroxysmal AF: Results of the TactiCath Contact Force Ablation Catheter Study for AF (TOCCASTAR) Study. Circulation 2015;132(10):907-15.

29. Natale A, Reddy VY, Monir G, et al. Paroxysmal AF catheter ablation with a contact force sensing catheter: results of the prospective, multicenter SMART-AF trial. J Am Coll Cardiol 2014;64(7):647-56.

30. Calkins H, Reynolds MR, Spector P, et al. Treatment of AF with antiarrhythmic drugs or radiofrequency ablation: two systematic literature reviews and meta-analyses. Circ Arrhythm Electrophysiol 2009;2(4):349-61.

31. Andrade JG, Khairy P, Dubuc M. Catheter cryoablation: biology and clinical uses. Circ Arrhythm Electrophysiol 2013;6(1):218-27.

32. Pandya B, Sheikh A, Spagnola J, et al. Safety and efficacy of secondgeneration versus first-generation cryoballoons for treatment of AF: a metaanalysis of current evidence. J Interv Card Electrophysiol 2015.

33. Andrade JG, Khairy P, Guerra PG, et al. Efficacy and safety of cryoballoon ablation for AF: a systematic review of published studies. Heart Rhythm 2011;8(9):1444-51.

34. Kuck KH, Brugada J, Furnkranz A, et al. Cryoballoon or Radiofrequency Ablation for Paroxysmal AF. N Engl J Med 2016;374(23):2235-45.

35. Buiatti A, von Olshausen G, Barthel P, et al. Cryoballoon vs. radiofrequency ablation for paroxysmal AF: an updated meta-analysis of randomized and observational studies. Europace 2017;19(3):378-84.

36. Andrade JG, Deyell MW, Badra M, et al. Randomized clinical trial of cryoballoon versus irrigated radio frequency catheter ablation for atrial fibrillation-the effect of double short versus standard exposure cryoablation duration during pulmonary vein isolation (CIRCA-DOSE): methods and rationale. BMJ Open 2017;7(10):e017970.

37. Padfield GJ, Steinberg C, Swampillai J, et al. Progression of paroxysmal to persistent AF: 10-year follow-up in the Canadian Registry of AF. Heart Rhythm 2017;14(6):801-7.

38. Cosedis Nielsen J, Johannessen A, Raatikainen P, et al. Radiofrequency ablation as initial therapy in paroxysmal AF. N Engl J Med 2012;367(17):1587-95. 
39. Morillo CA, Verma A, Connolly SJ, et al. Radiofrequency ablation vs antiarrhythmic drugs as first-line treatment of paroxysmal AF (RAAFT-2): a randomized trial. JAMA 2014;311(7):692-700.

40. Ball J, Carrington MJ, McMurray JJ, et al. AF: profile and burden of an evolving epidemic in the 21st century. Int J Cardiol 2013;167(5):1807-24.

41. Chugh SS, Havmoeller R, Narayanan K, et al. Worldwide epidemiology of AF: a Global Burden of Disease 2010 Study. Circulation 2014;129(8):837-47.

42. Ahmed MI, White M, Ekundayo OJ, et al. A history of AF and outcomes in chronic advanced systolic HF: a propensity-matched study. Eur Heart J 2009;30(16):2029-37.

43. Cha YM, Redfield MM, Shen WK, et al. AF and ventricular dysfunction: a vicious electromechanical cycle. Circulation 2004;109(23):2839-43.

44. Maisel WH, Stevenson LW. AF in HF: epidemiology, pathophysiology, and rationale for therapy. Am J Cardiol 2003;91(6A):2D-8D.

45. Mamas MA, Caldwell JC, Chacko S, Garratt CJ, Fath-Ordoubadi F, Neyses L. A meta-analysis of the prognostic significance of AF in chronic HF. Eur J Heart Fail 2009;11(7):676-83.

46. Santhanakrishnan R, Wang N, Larson MG, et al. AF Begets HF and Vice Versa: Temporal Associations and Differences in Preserved Versus Reduced Ejection Fraction. Circulation 2016;133(5):484-92.

47. Carlsson J, Miketic S, Windeler J, et al. Randomized trial of rate-control versus rhythm-control in persistent AF: the Strategies of Treatment of AF (STAF) study. J Am Coll Cardiol 2003;41(10):1690-6.

48. Hohnloser SH, Kuck KH, Lilienthal J. Rhythm or rate control in atrial fibrillation--Pharmacological Intervention in AF (PIAF): a randomized trial. Lancet 2000;356(9244):1789-94.

49. Roy D, Talajic M, Nattel S, et al. Rhythm control versus rate control for AF and HF. N Engl J Med 2008;358(25):2667-77.

50. Van Gelder IC, Hagens VE, Bosker HA, et al. A comparison of rate control and rhythm control in patients with recurrent persistent AF. N Engl J Med 2002;347(23):1834-40.
51. Wyse DG, Waldo AL, DiMarco JP, et al. A comparison of rate control and rhythm control in patients with AF. N Engl J Med 2002;347(23):1825-33.

52. Di Biase L, Mohanty P, Mohanty S, et al. ablation versus amiodarone for treatment of persistent AF in patients with congestive HF and an implanted device: Results From the AATAC Multicenter Randomized Trial. Circulation 2016;133(17):1637-44.

53. Hunter RJ, Berriman TJ, Diab I, et al. A Randomized Controlled Trial of Catheter Ablation Versus Medical Treatment of AF in HF (The CAMTAF Trial). Circ Arrhythm Electrophysiol 2014;7(1):31-8.

54. Jones DG, Haldar SK, Hussain W, et al. A randomized trial to assess catheter ablation versus rate control in the management of persistent AF in HF. J Am Coll Cardiol 2013;61(18):1894-903.

55. Khan MN, Jais P, Cummings J, et al. Pulmonary-vein isolation for AF in patients with HF. N Engl J Med 2008;359(17):1778-85.

56. MacDonald MR, Connelly DT, Hawkins NM, et al. Radiofrequency ablation for persistent $\mathrm{AF}$ in patients with advanced $\mathrm{HF}$ and severe left ventricular systolic dysfunction: a randomized controlled trial. Heart 2011;97(9):740-7.

57. Prabhu S, Taylor AJ, Costello BT, et al. Catheter ablation versus medical rate control in AF and systolic dysfunction: The CAMERA-MRI Study. J Am Coll Cardiol 2017;70(16):1949-61.

58. Marrouche NF, Brachmann J, Andresen D, et al. Catheter ablation for AF with HF. N Engl J Med 2018;378(5):417-27.

59. Packer DL, Mark DB, Robb RA, et al. Catheter Ablation versus Antiarrhythmic Drug Therapy for AF (CABANA) Trial: Study Rationale and Design. Am Heart J 2018;199:192-9.

60. Lau DH, Schotten U, Mahajan R, et al. Novel mechanisms in the pathogenesis of AF: practical applications. Eur Heart J 2016;37(20):1573-81. 